QUANTUM PROBABILITY

BANACH CENTER PUBLICATIONS, VOLUME 73

INSTITUTE OF MATHEMATICS

POLISH ACADEMY OF SCIENCES

WARSZAWA 2006

\title{
SPEED OF THE BROWNIAN LOOP ON A MANIFOLD
}

\author{
RÉMI LÉANDRE \\ Institut de Mathématiques de Bourgogne, Faculté des Sciences \\ 21000 Dijon, France \\ E-mail:remi.leandre@u-bourgogne.fr
}

\begin{abstract}
We define the speed of the curved Brownian bridge as a white noise distribution operating on stochastic Chen integrals.
\end{abstract}

1. Introduction. Let us consider the Brownian motion over $R: s \rightarrow B_{s}$. It is only continuous. Nevertheless, there are a lot of reasons to consider it as a Gaussian measure on the set of maps $\gamma:[0,1] \rightarrow R$

$$
d \mu=\frac{1}{Z} \exp \left[-\int_{0}^{1}|d / d s \gamma(s)|^{2} d s\right] d D(\gamma)
$$

where $d D(\gamma)$ is the formal Lebesgue measure on the set of paths.

This shows that it is interesting to define the speed of the Brownian path $d / d s B(s)$.

There are several approaches to defining the speed of the Brownian motion:

- The first one is Hida Calculus. The speed of the flat Brownian path is defined as a distribution on the space of Hida functionals ([Hi], [H.K.P.S], [O]). The symmetric Fock space plays a big role in this theory.

- The stochastic Chen-Souriau Calculus is the second one. Léandre has replaced the Brownian motion by the free loop space endowed with the B-H-K measure $\left([\mathrm{H}-\mathrm{K}],\left[\mathrm{Bi}_{1}\right]\right)$. Léandre ([ $\left.\left.\mathrm{L}_{5}\right],\left[\mathrm{L}_{6}\right]\right)$ has defined the canonical Killing vector field generating the canonical circle action on the continuous free loop space by using Chen-Souriau Calculus.

Our goal in this note is to define the speed $d / d s \gamma(s)$ of the Brownian bridge on a Riemannian compact manifold of dimension $d$, in the framework of Hida Calculus. We use the idea of Getzler ([Ge]) to do a current theory on the loop space by using Chen forms. By replacing Wiener chaos by Chen forms, Léandre $\left(\left[\mathrm{L}_{7}\right],\left[\mathrm{L}_{8}\right]\right)$ ) has defined a Feynman path integral on a manifold and has, in part, given a rigorous interpretation of

2000 Mathematics Subject Classification: Primary 58J65; Secondary 60H40.

Key words and phrases: speed of the curved Brownian bridge.

The paper is in final form and no version of it will be published elsewhere. 
the considerations of Atiyah-Witten-Bismut about the relation between the structure of the loop space and index theory ([At], $\left.\left[\mathrm{Bi}_{1}\right],\left[\mathrm{Bi}_{2}\right],\left[\mathrm{Bi}_{3}\right]\right)$.

We consider as in $\left[\mathrm{L}_{7}\right]$ a Fock space associated to smooth 1-forms on the manifold, the only difference with $\left[\mathrm{L}_{7}\right]$ is that we consider the interacting Fock space of AccardiBożejko (See $[$ A.B]). We consider the stochastic Chen iterated integrals map of JonesLéandre ([J.L]), which associate to some Fock space a set of functionals $F$ over the based Brownian bridge dense in $L^{p}, 1 \leq p<\infty$. If $G$ is a functional on the Brownian bridge, we can assimilate $G$ to the map

$$
F \rightarrow E[F G]
$$

We consider a 1-form on $\omega$ on $M$ and the functional $G_{t}=\int_{0}^{t}\langle\omega(\gamma(s)), d \gamma(s)\rangle$ on the Brownian bridge (we consider a Stratonovitch integral). Our two main results are:

Theorem A. $\mu_{t}: F \rightarrow E\left[F G_{t}\right]$ defines an Hida distribution.

TheOrem B. $d / d t \mu_{t}$ is an Hida distribution.

This explains that $\langle\omega(\gamma(t)), d / d t \gamma(t)\rangle$ is a Hida distribution on the Brownian bridge. In order to show Theorem B, we use shuffle products on Chen forms as well as some small time asymptotics on the Brownian bridge. For that, we use Malliavin Calculus technics. We refer to the surveys of Léandre $\left(\left[\mathrm{L}_{1}\right]\right)$, Kusuoka $([\mathrm{Ku}])$ and Watanabe $([\mathrm{Wa}])$ about this topic.

2. Fock space and stochastic Chen forms. Let $M$ be a compact Riemannian manifold with generic element $x$. Let $\Omega$ be the space of continuous functions from $[0,1]$ equal to 0 at 0 and at 1 into the space of 1 -forms on $M$. Let us denote such a form by $\omega_{t}$.

We consider on $\Omega$ variable Hilbert structures. Let $d d^{*}+d^{*} d=\Delta$ be the Laplacian acting on $\omega_{t}, t$ being frozen. And let $\nabla_{t}^{2}$ be the square of the covariant derivatives in $t$. Let $\Delta_{t o t}=-\nabla_{t}^{2}+\Delta$. We define:

$$
\|\left.\omega\right|_{k} ^{2}=\int_{0}^{1} d t \int_{M}\left\langle\left(\Delta_{t o t}+2\right)^{k} \omega(t), \omega(t)\right\rangle d m_{M}
$$

where $d m_{M}$ is the Riemannian measure on $M$. We get a Hilbert-Sobolev $H_{k}$ space conveniently complexified. We consider the interacting Fock space of Accardi-Bożejko $\Lambda_{k, C}$ of $H_{k}$ (See $\left.[\mathrm{A} . \mathrm{B}]\right)$. It is the space of

$$
\tilde{\sigma}=\sum \tilde{\sigma}_{n}
$$

where $\tilde{\sigma}_{n} \in\left(H_{k}\right)^{\otimes n}$ where we take the Hilbert norm:

$$
\|\tilde{\sigma}\|_{k, C}^{2}=\sum C^{n}\left\|\tilde{\sigma}_{n}\right\|_{H_{k}^{\otimes n}}^{2} n !
$$

Definition 2.1. The space $S_{\infty-}$ of white noise functionals is $\cap \Lambda_{k, C}$. Its dual $S_{-\infty}$ is called the space of Hida distributions.

An example of an element of the space of Hida functionals is a coherent vector:

$$
\exp [\omega]=\sum \frac{\omega^{n}}{n !}
$$


where $\omega$ is smooth on $\left[0,1 \times M\right.$. Let $\sigma_{n}=\frac{\omega_{1} \otimes \omega_{2} \cdots \otimes \omega_{n}}{n !}$. We consider

$$
F\left(\sigma_{n}\right)=\int_{0<s_{1}<\cdots<s_{n}<1}\left\langle\omega\left(s_{1}, \gamma\left(s_{1}\right)\right), d \gamma\left(s_{1}\right)\right\rangle \cdots\left\langle\omega\left(s_{n}, \gamma\left(s_{n}\right)\right), d \gamma\left(s_{n}\right)\right\rangle
$$

where $s \rightarrow \gamma(s)$ is the Brownian bridge starting from $x$ and arriving at $x$ time $s=1$. $d \gamma(s)$ is the Stratonovitch differential.

If we consider the heat kernel $p_{t}(x, y)$ associated to the Laplace-Beltrami operator on $M$, the law of the Brownian bridge $d P$ is the unique measure on the continuous loop space satisfying the following requirement: let $0 \leq s_{1}<s_{2}<\cdots<s_{n} \leq 1$ and $\gamma \rightarrow F\left(\gamma\left(s_{1}\right), \ldots, \gamma\left(s_{n}\right)\right)$ be a cylindrical functional ( $F$ is smooth from $M^{n}$ into $R$ ). We have:

$$
\begin{gathered}
E\left[F\left(\gamma\left(s_{1}\right), \ldots, \gamma\left(s_{n}\right)\right)\right]=\frac{1}{p_{1}(x, x)} \\
\int_{M^{n}} p_{s_{1}}\left(x, y_{1}\right) p_{s_{2}-s_{1}}\left(y_{1}, y_{2}\right) \ldots p_{1, s_{n}}\left(y_{n}, x\right) F\left(y_{1}, \ldots, y_{n}\right) d m_{M}\left(y_{1}\right) \cdots d m_{M}\left(y_{n}\right) .
\end{gathered}
$$

Moreover, $s \rightarrow \gamma(s)$ is a semi-martingale. We refer to [El], [I.W] and [Ma $]$ for material about diffusion processes on manifolds.

By using Sobolev's imbedding theorem, let us recall the following fact ([J.L], $\left[\mathrm{L}_{2}\right]$ ) called by Jones-Léandre Schwartz's lemma ([Sc]):

$$
E\left[\left|F\left(\sigma_{n}\right)\right|^{p}\right]^{1 / p} \leq \frac{C^{n}}{\sqrt{n !}} \prod\left\|\omega_{i}\right\|_{k}
$$

for some big $k$. As a matter of fact, Jones-Léandre used another space: they consider the Levi-Civita connection on $S^{1} \times M \nabla$. If $\omega(s, y)$ is considered as a 1-form on $S^{1} \times M$, Jones-Léandre consider:

$$
\|\omega\|_{k, \infty}=\|\omega\|+\|\nabla \omega\|_{\infty}+\cdots+\left\|\nabla^{k} \omega\right\|_{\infty}
$$

and show the estimate:

$$
E\left[\left|F\left(\sigma_{n}\right)\right|^{p}\right]^{1 / p} \leq \frac{C^{n}}{\sqrt{n !}} \prod\left\|\omega_{i}\right\|_{k, \infty}
$$

But we have $([\mathrm{Gi}])$ :

$$
\|\omega\|_{k, \infty} \leq C\|\omega\|_{k^{\prime}}
$$

Let $\omega_{j}$ be an orthonormal basis common to each $H_{k}$. The $\omega_{j}$ are of $L^{2}$ norm 1 . Let $J=\left(i_{1}, \ldots, i_{|J|}\right)$ and let

$$
\omega_{J}=\frac{\omega_{i_{1}} \otimes \cdots \otimes \omega_{i_{|J|}}}{|J| !}
$$

We write:

$$
\left\|\omega_{J}\right\|_{k}^{2}=\prod\left\|\omega_{i_{j}}\right\|_{k}^{2}
$$

If $\tilde{\sigma}=\sum \lambda_{J} \omega_{J}$, we get by (2.7)

$$
E\left[|F(\tilde{\sigma})|^{p}\right]^{1 / p} \leq \sum\left|\lambda_{J}\right| \frac{C^{|J|}}{\sqrt{|J| !}}\left\|\omega_{J}\right\|_{k}
$$


$\omega_{j}$ is associated to the eigenvalue $\lambda_{j}$ of $\Delta_{t o t}+2$. We use the Cauchy-Schwarz inequality in order to get:

$$
E\left[|F(\tilde{\sigma})|^{p}\right]^{1 / p} \leq C\|\tilde{\sigma}\|_{k^{\prime}, C^{\prime}}\left(\sum_{J} C_{1}^{|J|} \prod_{i_{j} \in J}\left(\lambda_{i_{j}}^{-k_{1}}\right)\right)^{1 / 2}
$$

for some big $k^{\prime}$, some big $C^{\prime}$ and some small $C_{1}$ and some big $k_{1}$. We arrange the $\lambda_{j}$ 's in increasing order. Let us recall (see [Gi]) that $\lambda_{j} \sim C j^{r}$ for some $r>0$. We deduce that the constant in the right hand side of (2.13) is finite because it is equal to:

$$
\sum_{n} C_{1}^{n}\left(\sum \lambda_{i}^{-k_{1}}\right)^{n}<\infty
$$

if $k_{1}$ is big enough and $C_{1}$ small enough. We deduce:

TheOREM 2.1. The Chen iterated integral map F maps continuously $S_{\infty-}$ into $L^{p}$.

Moreover:

TheOREM 2.2. $F\left(S_{\infty-}\right)$ is dense in $L^{p}$.

Proof. If $g$ is a smooth function on $M$ then

$$
g(\gamma(t))-g(\gamma(0))=\int_{0}^{t}\langle d g(\gamma(s)), d \gamma(s)\rangle
$$

This yields the result.

REMARK 2.1. If the forms $\omega$ in the definition of the stochastic Chen form don't depend on $t$, Theorem 2.2 is not true (but in cohomology, the result remains true: see $\left[\mathrm{L}_{4}\right]$ for a stochastic version of this classical result of Adams in algebraic topology [Ch]).

Our basic idea for the following is to identify a random variable $(G)$ in $L^{2}$ to the map defined on $S_{\infty-}$

$$
\tilde{\sigma} \rightarrow E[G F(\tilde{\sigma})]=\mu(G)(\tilde{\sigma}) .
$$

By Theorem 2.2, this identification is consistent.

\section{Shuffle product and theorem A}

Theorem A. $\mu(G)$ the linear map which associates $E[G F(\tilde{\sigma})]$ to $\tilde{\sigma}$ belonging to $S_{\infty-}$ defines a white noise distribution.

Proof. We have by Hölder inequality for some $p$ :

$$
\mu(G)(\tilde{\sigma}) \leq C E\left[F^{p}(\tilde{\sigma})\right]^{1 / p} \leq C\|\tilde{\sigma}\|_{k, C}
$$

for some $k$ and $C$. This gives the result.

If $G_{t}=\int_{0}^{t}\langle\omega(\gamma(s)), d \gamma(s)\rangle$, we can express $\mu\left(G_{t}\right)$ by using the coproduct on Chen forms $([\mathrm{Ch}])$ and shuffle product. Let us consider $\omega_{J}$ as in the previous part, the normalizing factorial being excluded. We associate $\sum_{I} F_{t, I}^{1}\left(\omega_{J}\right) F_{t, I}^{2}\left(\omega_{J}\right)$ where $I=\left\{i_{1}, i_{2}, \ldots, i_{|I|}\right\}$ and $I^{c}=\left\{i_{|I|+1}, \ldots, i_{|J|}\right\}$. Moreover,

$$
F_{t, I}^{1}\left(\omega_{J}\right)=\int_{0}^{t}\left\langle\omega_{i_{1}}\left(s_{1}, \gamma\left(s_{1}\right)\right), d \gamma\left(s_{1}\right)\right\rangle \cdots\left\langle\omega_{i_{|I|}}\left(s_{|I|}, \gamma\left(s_{|I|}\right)\right), d \gamma\left(s_{|I|}\right)\right\rangle
$$


and

$$
\begin{gathered}
F_{t, I}^{2}\left(\omega_{J}\right)=\int_{t<s_{|I|+1}<\cdots<s_{|J|}<1} \\
\left\langle\omega_{i_{|I|+1}}\left(s_{|I|+1}, \gamma\left(s_{|I|+1}\right)\right), d \gamma\left(s_{|I|+1}\right)\right\rangle \cdots\left\langle\omega_{i_{|J|}}, \gamma\left(s_{|J|}\right), d \gamma\left(s_{|J|}\right)\right\rangle .
\end{gathered}
$$

It is the habitual coproduct for Chen iterated integrals ([Ch]): we use Stratonovitch integrals. Let us recall that we have used integrals on the simplex, because we did not divide by $|J|$ ! as in (2.8). $F_{t, I}^{1}\left(\omega_{J}\right)$ is an integral on the simplex $0<s_{1}<\cdots<s_{|I|}<t$. We consider the product $G_{t} F_{t, I}^{1}\left(\omega_{J}\right)$ as a sum over all segments $I_{1}=\left\{i_{1}, i_{2}, \ldots, i_{I_{1}}\right\}$ included in $I$ of the iterated integrals

$$
\begin{gathered}
\int_{0<s_{1}<s_{2}<\cdots<s_{\left|I_{1}\right|}<s<s_{\left|I_{1}\right|+1}<\cdots<s_{|I|}<t}\left\langle\omega_{i_{1}}\left(s_{1}, \gamma\left(s_{1}\right)\right), d \gamma\left(s_{1}\right)\right\rangle \cdots \\
\cdot\left\langle\omega_{i_{\left|I_{1}\right|} \mid}\left(s_{\left|I_{1}\right|}, \gamma\left(s_{\left|I_{1}\right|}\right)\right), d \gamma\left(s_{\left|I_{1}\right|}\right)\right\rangle \cdots \\
\cdot\langle\omega(\gamma(s)), d \gamma(s)\rangle\left\langle\omega_{i_{\left|I_{1}\right|+1}}\left(s_{\left|I_{1}\right|+1}, \gamma\left(s_{\left|I_{1}\right|+1}\right)\right), d \gamma\left(s_{\left|I_{1}\right|+1}\right)\right\rangle \\
\left.\cdots\left\langle\omega_{i_{|I|}}\left(s_{|I|}\right), \gamma\left(s_{|I|}\right)\right), d \gamma\left(s_{|I|}\right)\right\rangle=G_{t, I_{1}, I}\left(\omega_{J}\right) .
\end{gathered}
$$

It is the traditional shuffle product of Chen iterated integrals ([Ch]). We get formally:

LEMMA 3.1. We have the following decomposition formula:

$$
G_{t} F\left(\omega_{J}\right)=\sum_{I_{1} \subseteq I \subseteq J} G_{t, I_{1}, I}\left(\omega_{J}\right) F_{t, I}^{2}\left(\omega_{J}\right) .
$$

4. Malliavin Calculus and theorem B. By Lemma 3.1,

$$
\mu_{t}\left(F(\tilde{\sigma})=\sum_{J} \lambda_{J} \sum_{I_{1} \subseteq I \subseteq J} E\left[G_{t, I_{1}, I}\left(\omega_{J}\right) F_{t, I}^{2}\left(\omega_{J}\right)\right] .\right.
$$

Let us suppose that $t \in] 0,1[$. We gave the following lemma got by Malliavin Calculus.

LEMma 4.1. $\frac{\partial}{\partial t} E\left[G_{t, I_{1}, I}\left(\omega_{J}\right) F_{t, I}^{2}\left(\omega_{J}\right)\right]$ is smaller than $\frac{C^{|J|}}{(|I| !(|J|-|I|) !)^{1 / 2}} \prod_{i_{j} \in J}\left\|\omega_{i_{J}}\right\|_{k}$ for some $k$ and some $C$.

Proof. We condition in $E\left[G_{t, I_{1}, I}\left(\omega_{J}\right) F_{t, I}^{2}\left(\omega_{J}\right)\right]$ by $\gamma(t)$. Let $q_{t}(y)$ be the density of the law of $\gamma(t)$ : it depends smoothly from $t \in] 0,1\left[\right.$ and $y$. Let $P_{s, y, z}$ be the law of the Brownian bridge starting from $y$ and arriving at time $s$ at $z$. By proceeding as in [J.L], we get:

$$
E\left[G_{t, I_{1}, I}\left(\omega_{J}\right) F_{t, I}^{2}\left(\omega_{J}\right)\right]=\int_{M} q_{t}(y) d m_{M}(y) E_{t, x, y}\left[G_{t, I_{1}, I}\left(\omega_{J}\right)\right] E_{1-t, y, x}\left[F_{t, I}^{2}\left(\omega_{J}\right)\right] .
$$

We have to estimate $\frac{\partial}{\partial t} E_{t, x, y}\left[G_{t, I_{1}, I}\left(\omega_{J}\right)\right]$ and $\frac{\partial}{\partial t} E_{1-t, y, x}\left[F_{t, I}^{2}\left(\omega_{J}\right)\right]$. Since $\left.t \in\right] 0,1[$, the considerations leading to the estimate of each term are very similar. Let us do the first one.

We put $t=\epsilon^{2}$ following Molchanov $([\mathrm{Mo}])$ and we consider the Brownian motion $\gamma(t)$ between 0 and $t$ as the Brownian motion $\gamma(s)(\epsilon)$ between 0 and 1 , where $\gamma(s)(\epsilon)$ is given by the Eells-Elworthy-Malliavin equation:

$$
d \gamma(s)(\epsilon)=\epsilon \tau(s)(\epsilon) d B(s)
$$

where $\tau(s)(\epsilon)$ is the parallel transport along the path $u \rightarrow \gamma(u)(\epsilon)$ for the Levi-Civita 
connection on the Riemannian manifold $M$ and $B(s)$ is a Brownian motion in the tangent space at $x$ of the Riemannian manifold (see $\left[\mathrm{Bi}_{1}\right]$ and $\left[\mathrm{L}_{1}\right]$ for this statement).

$G_{t, I_{1}, I}\left(\omega_{J}\right)$ is transformed into $\epsilon \rightarrow G_{\epsilon, I_{1}, I}\left(\omega_{J}\right)$ and is almost surely smooth in $\epsilon$. Moreover $\frac{d^{r}}{d \epsilon^{r}} G_{\epsilon, I_{1}, I}\left(\omega_{J}\right)$ is bounded in all the Sobolev spaces of Malliavin Calculus. We consider the measure $\nu_{\epsilon}$ :

$$
f \rightarrow E\left[G_{\epsilon, I_{1}, I}\left(\omega_{J}\right) f(\gamma(1)(\epsilon))\right]
$$

It has a density smooth in $\epsilon>0$, by using Malliavin Calculus technics (see the surveys $\left[\mathrm{L}_{1}\right],[\mathrm{Ku}]$ and $\left.[\mathrm{Wa}]\right)$. Moreover the derivative in $\epsilon>0$ of the density of $\nu_{\epsilon}$ is smaller than $\frac{C^{|I|}}{\sqrt{|I| !}} \prod_{i_{j} \in I}\left\|\omega_{i_{j}}\right\|_{k}$ for some $k$ (see $\left.\left[\mathrm{L}_{1}\right],\left(\mathrm{L}_{2}\right]\right)$. But this density is nothing else than

$$
E\left[G_{\epsilon, I_{1}, I}\left(\omega_{J}\right) \mid \gamma(1)(\epsilon)=y\right] p_{\epsilon^{2}}(x, y)
$$

and $p_{\epsilon^{2}}(x, y)$ has bounded derivatives in $\epsilon>0$.

ThEOREM B.1. If $t \in] 0,1\left[, \frac{\partial}{\partial t} \mu_{t}\right.$ is a white noise distribution.

Proof. We have:

$$
\frac{\partial}{\partial t} \mu_{t}(F(\tilde{\sigma}))=\sum_{J} \lambda_{J} \sum_{I_{1} \subseteq I \subseteq J} \frac{\partial}{\partial t} E\left[G_{t, I_{1}, I}\left(\omega_{J}\right) F_{t, I}^{2}\left(\omega_{J}\right)\right] .
$$

In absolute value, it is smaller than:

$$
\sum_{J}\left|\lambda_{J}\right| \frac{C^{|J|}}{\sqrt{|I| !}} \prod_{i_{j} \in J}\left\|\omega_{i_{j}}\right\|_{k}
$$

We conclude as in Theorem 2.1.

We would like to show that Theorem B is still true for $t=0$ (or equivalently in $t=1$ ).

We remark that $E\left[\left|G_{t, I_{1}, I}\right|\right]=o(t)$ when $t \rightarrow 0$ if $I$ has a length larger than 2. So it is enough to consider the case where $I$ is reduced to a singleton or to the empty set. Moreover, if $y$ and $x$ are not close, we have in short time $p_{t}(x, y) \leq \exp [-C / t]$ for some $C>0$. Let us do the asymptotic expansion of the density of the measure $\nu_{\epsilon}$ when $\epsilon \rightarrow 0$. By the previous considerations, we can work in normal coordinates around $x$. We do the following rescaling in normal coordinates, $\gamma(1)(\epsilon) \rightarrow \frac{\gamma(1)(\epsilon)-x}{\epsilon}$ which tends when $\epsilon \rightarrow 0$ in all the Sobolev spaces of Malliavin Calculus to a non-degenerate Gaussian variable on $T_{x}(M)$. But we are interested in the asymptotic expansion for $y \neq x$. In order to simplify the exposition, we consider the Brownian motion written in Schwartz form in a neighborhood of $x$

$$
d \gamma(s)(\epsilon)=\epsilon \sum_{i=1}^{d} X_{i}(\gamma(s)(\epsilon)) d B_{s}^{i}+\epsilon^{2} X_{0}(\gamma(s)(\epsilon)) d s
$$

for some smooth vector fields in the neighborhood of $x$. We consider, if $x$ and $y$ are close, the equation of the unique geodesic joining $x$ to $y$

$$
d \gamma(s)(0, h)=\sum_{i=1}^{d} X_{i}(\gamma(s)(0, h)) h_{s}^{i} d s
$$

We do the translation $\epsilon d B \rightarrow \epsilon d B+d h$ for the previous $h$ ( $x$ and $y$ are close). 
$G_{\epsilon, I_{1}, I}\left(\omega_{J}\right)$ is replaced by $\tilde{G}_{I_{1}, I}\left(\omega_{J}\right)(\epsilon d B+d h)$ and we get a Cameron-Martin-GirsanovMartin term in $\exp \left[-d^{2}(x, y) / 2 \epsilon^{2}\right] \exp \left[-\left\langle h_{1}, B_{1}\right\rangle / \epsilon\right] \cdot \gamma(1)(\epsilon)$ is replaced by $\gamma(1)(\epsilon, h)$ such that $\gamma(1)(0, h)=y$ and such that $\frac{\partial}{\partial \epsilon} \gamma(1)(0, h)=0$ implies $\int_{0}^{1}\left\langle h_{s}, d B_{s}\right\rangle=0\left(\left[\mathrm{~L}_{1}\right]\right)$. After rescaling $\gamma(1)(\epsilon, h)$ by $\frac{\gamma(\epsilon, h)-y}{\epsilon}$, we get an asymptotic expansion of the density of $\nu_{\epsilon}$ where only even powers of $\epsilon$ remain, because the expectation of an odd expression for a Gaussian measure of average 0 is equal to 0 .

More precisely, if $I=i_{0}$, we get the asymptotic expansion of the density of $\nu_{\epsilon}$ in

$$
\epsilon^{-d}\left(B_{0}(y)+\epsilon^{2} B_{1}(y)+O\left(\epsilon^{4}\right)\right) \exp \left[-\frac{d^{2}(x, y)}{2 \epsilon^{2}}\right]
$$

where $d^{2}(x, y)$ is the Riemannian distance between $x$ and $y, d$ the dimension of the manifold and $B_{0}(y)=\tilde{G}_{I_{1}, I}\left(\omega_{J}\right)(d h)$ is a quadratic expression when $y \rightarrow x$. If we replace $\epsilon^{2}$ by $t$, we get an asymptotic expansion

$$
c_{t}(x, y)=t^{-d / 2}\left(B_{0}(y)+t B_{1}(y)+O\left(t^{2}\right)\right) \exp \left[-\frac{d^{2}(x, y)}{2 t}\right] .
$$

The integral of the term in $B_{0}(y)$ tends to 0 when $t \rightarrow 0$, because $B_{0}(y)$ is quadratic and behaves in $t C(x)$ after doing the rescaling in the Gaussian integral of $y$ in $\sqrt{t} y$, and we deduce that the time derivative in $t=0$ is given by $B_{1}(x)+C(x)$.

If $I=\emptyset$, we do the asymptotic expansion of $B_{0}(y)$. The boring term, which cannot be handled by the previous consideration, is $\langle\omega(x), y\rangle$. We have to compute the time derivative in $t=0$ of

$$
\int_{B}\langle\omega(x), y\rangle \exp \left[-\frac{|y|^{2}}{2 t}\right] d y
$$

for a compact neighborhood of 0 in $T_{x}(M)$, because $d(x, y)=|y|$ in normal coordinates ( $x$ is assimilated to 0 in normal coordinates). But this time the derivative is trivially 0.

This shows us that:

$$
\left|\frac{\partial}{\partial t} E\left[G_{t, I_{1}, I}\left(\omega_{J}\right) F_{t, I}^{2}\left(\omega_{J}\right)\right]\right|_{t=0} \mid \leq \frac{C^{|I|}}{\sqrt{|I| !}} \prod_{i_{j} \in J}\left\|\omega_{i_{j}}\right\|_{k} .
$$

We conclude as before that:

TheOREM B.2. $\frac{\partial}{\partial t} \mu_{t}$ is a white noise distribution at $t=0$ and $t=1$.

REMARK 4.1. We could replace $G_{t}$ by $\int_{0}^{t}\langle\omega(s, \gamma(s)), d \gamma(s)\rangle$. This leads to some consistency problem. If $\omega(t,)=$.0 in $t$, the time derivative in $t$ of $\mu_{s}$ is equal to 0 . Namely, in such a case, we have for all $p>1$

$$
\left|E\left[\left(G_{t+\Delta t}-G_{t}\right) F(\tilde{\sigma})\right]\right| \leq(\Delta t)^{3 / 2}\|F(\tilde{\sigma})\|_{L^{p}} .
$$

This yields the result.

\section{References}

[A.B] L. Accardi and M. Bożejko, Interacting Fock spaces and Gaussianization of probability measures, Inf. Dim. Ana. Quant. Probab. Rel. Top. 1 (1998), 663-670. 
[At] M. Atiyah, Circular symmetry and stationary phase approximation, in: Colloque en l'honneur de L. Schwartz (Paris, 1983), Astérisque 131 (1985), 43-59.

[B] Y. M. Berezansky, Infinite dimensional non-Gaussian analysis connected with generalized translation operators, in: Analysis on Infinite-Dimensional Lie Groups and Algebras (Marseille, 1997), H. Heyer and J. Marion (eds.), World Scientific, Singapore, 1999, 22-46.

[B.K] Y. M. Berezansky and Y. G. Kondratiev, Spectral Methods in Infinite Dimensional Analysis, Vol. 1,2, Kluwer, Dordrecht, 1995.

[Bi $\left.{ }_{1}\right] \quad$ J. M. Bismut, Index theory and equivariant cohomology of the loop space, Com. Math. Phys. 98 (1985), 213-237.

$\left[\mathrm{Bi}_{2}\right] \quad$ J. M. Bismut, Localization formula, superconnections and the index theorem for families, Com. Math. Phys. 103 (1986), 91-151.

[Bi $\left.{ }_{3}\right]$ J. M. Bismut, Filtering equation, equivariant cohomology and the Chern character, in: VIIIth International Congress of Mathematical Physics (Marseille, 1986), R. Sénéor (ed.), World Scientific, Singapore, 1987, 17-56.

[Ch] K. T. Chen, Iterated path integrals of differential forms and loop space homology, Ann. Maths. 97 (1973), 213-237.

[El] K. D. Elworthy, Stochastic Differential Equations on Manifolds, L.M.S. Lectures Notes Series 20, Cambridge University Press, Cambridge, 1982.

[Ge] E. K. Getzler, Cyclic homology and the path integral of the Dirac operator, preprint, Harvard University, 1988.

[Ge.J.P] E. Getzler, J. D. S. Jones and S. Petrack, Differential forms on a loop space and the cyclic bar complex, Topology 30 (1991), 333-373.

[Gi] P. Gilkey, Invariance Theory, the Heat Equation and the Atiyah-Singer Theorem, C.R.C. Press, Baton Rouge, 1995.

[Hi] T. Hida, Analysis of Brownian Functionals, Carleton Math. Lect. Notes 13, Ottawa, 1975.

[H.K] R. Hoegh-Krohn, Relativistic quantum statistical mechanics in 2 dimensional space time, Com. Math. Phys. 38 (1974), 195-224.

[I.W] N. Ikeda and S. Watanabe, Stochastic Differential Equations and Diffusion Processes, North-Holland, Amsterdam, 1981.

[H.K.P.S] T. Hida, H. H. Kuo, J. Potthoff and L. Streit, White Noise: an Infinite Dimensional Calculus, Kluwer, Dordrecht, 1993.

[J.L] J. D. S. Jones and R. Léandre, $L^{p}$ Chen forms on loop spaces, in: Stochastic Analysis (Durham, 1990), M. Barlow and N. Bingham (ed.), Cambridge University Press, Cambridge, 1991, 104-162.

$[\mathrm{Ku}] \quad$ S. Kusuoka, More recent theory of Malliavin Calculus, Sugaku 5 (1992), 155-173.

$\left[\mathrm{L}_{1}\right] \quad$ R. Léandre, Appliquations quantitatives et qualitatives du Calcul de Malliavin, in: Col. Franco-Japonais (Paris, 1987), M. Métivier and S. Watanabes (eds.), Lect. Notes Maths. 1322, Springer, Heidelberg, 1988, 109-133. English translation in: Geometry of Random Motion (Cornell, 1987), R. Durrett and M. Pinsky (eds.), Cont. Maths. 73, A.M.S., Providence, 1988, 173-197.

$\left[\mathrm{L}_{2}\right] \quad \mathrm{R}$. Léandre, Cohomologie de Bismut-Nualart-Pardoux et cohomologie de Hochschild entière, in: Séminaire de Probabilités XXX in honour of P. A. Meyer and J. Neveu, J. Azéma et al. (eds.), Lect. Notes. Math. 1626, Springer, Heidelberg, 1996, 68-100.

$\left[\mathrm{L}_{3}\right] \quad$ R. Léandre, Brownian cohomology of an homogeneous manifold, in: New Trends in Stochastic Analysis (Charingworth, 1994), K. D. Elworthy et al. (eds.), World Scientific, Singapore, 1997, 305-347. 
[L $\left.\mathrm{L}_{4}\right] \quad$ R. Léandre, Stochastic Adams theorem for a general compact manifold, Rev. Math. Phys. 13 (2001), 1095-1133.

[L5] R. Léandre, Stochastic equivariant cohomologies and cyclic cohomology, Ann. Probab., to appear.

$\left[\mathrm{L}_{6}\right] \quad$ R. Léandre, Hypoelliptic diffusion and cyclic cohomology, in: Stochastic analysis (Ascona 2002), R. Dalang et al. (eds.), Prog. Probab. 58, Birkhäuser, Basel, 2004, 165185.

$\left[\mathrm{L}_{7}\right] \quad$ R. Léandre, Theory of distribution in the sense of Connes-Hida and Feynman path integral on a manifold, Inf. Dim. Ana. Quant. Probab. Rel. Top. 6 (2003), 505-517.

$\left[\mathrm{L}_{8}\right] \quad \mathrm{R}$. Léandre, White noise analysis, filtering equation and the index theorem for families, in: Infinite Dimensional Harmonic Analysis (Tuebingen, 2003), H. Heyer and H. Saitô (eds.), to appear.

$\left[\mathrm{Ma}_{1}\right] \quad$ P. Malliavin, Stochastic calculus of variations and hypoelliptic operators, in: Stochastic analysis (Kyoto 1975), K. Itô (ed.), Kinokuniya, Tokyo, 1978, 155-263.

$\left[\mathrm{Ma}_{2}\right] \quad$ P. Malliavin, Stochastic Analysis, Springer, Heidelberg, 1998.

[Mo] S. Molchanov, Diffusion processes and Riemannian geometry, Russ. Math. Surveys 30 (1975), 1-63.

[Nu] D. Nualart, The Malliavin Calculus and Related Topics, Springer, Heidelberg, 1995.

[O] N. Obata, White Noise Analysis and Fock Space, Lect. Notes. Math. 1577, Springer, Berlin, 1990.

[Sc] L. Schwartz, La convergence de la série de Picard pour les équations différentielles stochastiques, in: Séminaire de Probabilités XXIII, J. Azéma et al. (eds.), Lect. Notes Math. 1372, Springer, Heidelberg, 1984, 343-355.

[Wa] S. Watanabe, Stochastic analysis and its application, Sugaku 5 (1992), 51-71. 
